\title{
Gradhiva
}

GRADHIV

Revue d'anthropologie et d'histoire des arts

$7 \mid 2008$

Le possédé spectaculaire

Gérald Bloncourt et Michael Löwy, Messagers de la tempête. André Breton et la révolution de janvier 1946 en Haïti

Pantin, Le Temps des Cerises, 2007, 181 p.

Andrea D'Urso

2 OpenEdition

Journals

Édition électronique

URL : http://journals.openedition.org/gradhiva/1152

DOI : 10.4000/gradhiva. 1152

ISSN : 1760-849X

Éditeur

Musée du quai Branly Jacques Chirac

Édition imprimée

Date de publication : 15 mai 2008

Pagination : 168-170

ISBN : 978-2-915133-86-8

ISSN : 0764-8928

Référence électronique

Andrea D'Urso, « Gérald Bloncourt et Michael Löwy, Messagers de la tempête. André Breton et la révolution de janvier 1946 en Haïti », Gradhiva [En ligne], 7| 2008, mis en ligne le 10 décembre 2008, consulté le 21 septembre 2020. URL : http://journals.openedition.org/gradhiva/1152 ; DOI : https:// doi.org/10.4000/gradhiva.1152

Ce document a été généré automatiquement le 21 septembre 2020.

(c) musée du quai Branly 


\section{Gérald Bloncourt et Michael Löwy, Messagers de la tempête. André Breton et la révolution de janvier 1946 en Haïti}

Pantin, Le Temps des Cerises, 2007, 181 p.

\section{Andrea D'Urso}

\section{RÉFÉRENCE}

Gérald Bloncourt et Michael Löwy, Messagers de la tempête. André Breton et la révolution de janvier 1946 en Haïti. Pantin, Le Temps des Cerises, 2007, 181 p.

1 Le récent livre de Gérald Bloncourt et Michael Löwy porte sur les événements des Cinq Glorieuses, les journées haïtiennes du 7 au 11 janvier 1946 qui entraînèrent la chute du dictateur Élie Lescot, et sur "cette fusion explosive entre poésie et insurrection, surréalisme et révolte sociale, [qui] est un cas peut-être unique dans l'histoire des révolutions modernes» (p. 10). Dans l'intention déclarée de «combler cette étrange lacune » après soixante ans (p. 11), ce livre présente le témoignage de la participation directe de Bloncourt (d'abord peintre, puis photographe, poète et écrivain lui-même) aux événements en question (p. 69-122) : c'est le souvenir de cet octogénaire qui garde dans son récit toute l'ardeur du jeune homme âgé de 19 ans qu'il était alors, faisant revivre, surtout dans l'esprit des jeunes justement, cette échelle émotionnelle des sentiments propres probablement à toutes les révolutions, spécialement si elles sont ratées.

2 On y trouvera, en outre, un remarquable recueil de documents, photographies, coupures de journaux, poésies et reproductions de tableaux qui ne sont pas uniquement signés par Bloncourt, mais aussi par ses camarades de lutte. Parmi ces derniers, nous nous bornons à rappeler René Depestre (qui a lui aussi beaucoup écrit sur ces événements) et Jacques Stephen Alexis, fondateurs de $L a$ Ruche, la revue où paraîtront certains textes des conférences d'André Breton que Bloncourt et Löwy 
considèrent comme de véritables « étincelles [qui] sont tombées sur une poudre sèche et inflammable : la rage du peuple haïtien et son désir de liberté » (p. 10).

De son côté, dans la première partie du livre, Michael Löwy s'interroge sur cette « "coöncidence" entre [...] l'éclosion de la révolte de janvier 1946 » et la visite de Breton (p. 24), qui avait été invité pendant son exil new-yorkais à tenir des conférences en Haïti par son cher ami, le surréaliste Pierre Mabille, qui se trouvait déjà sur l'île depuis 1941. C'est seulement grâce à ce dernier, l'un des rares Blancs qui soient admis aux rites vodou haïtiens, que Breton put accéder à ce monde de «forces primitives » dont il évoque le souveniren 1962 dans Pont-Levis (repris dans Perspective cavalière. Paris, Gallimard, 1970).

4 Le rôle de Mabille est rappelé par nos auteurs, car il avait d'abord aidé Bloncourt à échapper aux griffes de la police puis s'était engagé à commuer sa condamnation en exil. Löwy le rappelle justement au début du livre, dans l'intention de montrer l'intérêt du surréalisme, notamment celui de Breton, pour ce qu'il nomme la «constellation noire des Caraïbes » (p. 15). Ce qui lui semble typique de certains surréalistes, de Breton à Vincent Bounoure, est « une sorte d'anthropologie de la magie - qui est en même temps une anthropologie du désir -, qui leur permet de construire des vases communicants entre l'hermétisme, le romantisme, le surréalisme et les cultures dites "primitives" " (p. 20), lesquelles «ont gardé quelque chose de cette unité magique originaire, et qui ont réussi à résister à l'acide dissolvant de la valeur d'échange capitaliste » (p. 21).

5 Nous trouvons là une référence implicite au parcours illustré dans L'Art magique et à ces pouvoirs perdus dont parlait Breton et que Bounoure avec lui - utilisant la formule d'«anthropologie du désir», plus prudente et plus analytiquement développée que celle avancée par Löwy, " anthropologie de la magie » - considérait comme beaucoup moins réprimés dans les civilisations dites «sauvages". À cet égard, une citation de Bounoure ne serait pas hors de propos: "L'anthropologie est une érotologie : elle n'a pas de tâche plus urgente que de déterminer pratiquement, et d'abord dans son exercice même, les conditions dans lesquelles pourrait naître une civilisation ouverte à la satisfaction aussi bien qu'à l'expression entière du désir. Marquer ce lieu sur les cartes de l'esprit et le conquérir serait réinvestir l'homme de l'intégralité de ses pouvoirs et donner authentiquement au monde la parole. Ainsi se trouve conjurée la vérité de l'entreprise anthropologique. Si l'anthropologue fait enfin silence, c'est pour laisser place à la parole essentielle, celle qui éclate dans les phrases du poète, c'est-àdire de l'insurgé1.»

6 Pour revenir à la thèse principale de Löwy, tout en affirmant très clairement qu'il ne veut point exagérer l'importance du discours de Breton et tout en reconnaissant la limite de la comparaison entre la harangue charismatique des dirigeants révolutionnaires et celle « qui voit un poète s'adresser à un groupe de jeunes pour leur expliquer les aspirations émancipatrices du surréalisme » (p. 38), cet auteur reprend le concept d'« influence » comme l'utilisait son maître, Lucien Goldmann, c'est-à-dire en tant que « choix actif» des Haïtiens ayant trouvé chez Breton la parole radicale qu'ils cherchaient (p. 40), et il se déclare convaincu que «les interventions du poète surréaliste ont apporté quelque chose à la gestation - chez les étudiants, la jeunesse et certaines couches populaires plus cultivées - d'un certain état d'esprit, d'un climat, d'une atmosphère orageuse favorable à un grand élan émancipateur » (p. 26).

7 Il est également vrai que l'interprétation de Löwy semble correspondre parfois au ton des témoignages (reproduits dans les documents en appendice) de Depestre, qui parle 
du « magnétisme » charmant de Breton, de « son air de prophète » (p. 51), et d'un autre poète, Paul Laraque, qui parle de Breton comme «du Mage » (p. 57). Ainsi, pour Löwy, Breton n'est pas seulement «un des messagers de la tempête de janvier 1946 », mais même « un des sorciers qui, comme les hougans [prêtres] du vodou, ont le don sacré de prononcer les paroles enchantées qui déclenchent la foudre... » (p. 26).

8 Selon Löwy, il semblerait ainsi que le premier discours de Breton à l'hôtel Savoy, le 5 décembre 1945, plus tard publié dans le numéro spécial de La Ruche du $1^{\mathrm{er}}$ janvier 1946, qui sera saisi par la police, «a été une sorte de rendez-vous magique entre Breton et les poètes et la jeunesse haïtiennes » (p. 26), fondé sur « cet esprit surréaliste de sympathie - au sens étymologique du mot : un pathos partagé » (p. 20-21). La première conférence officielle, parmi les douze initialement prévues, prononcée le 20 décembre au théâtre Rex et parue le $1^{\mathrm{er}}$ janvier, mais cette fois dans la revue Conjonctions, qui n'a pas été saisie, "a peut-être contribué elle aussi à préparer l'ouragan tropical ", ajoute Löwy, grâce à la reprise de quelques thèmes déjà traités dans le discours précédent (comme la tradition révolutionnaire de l'île) et à l'évocation plus claire des sujets seulement effleurés ou absents auparavant, tels que la pauvreté du peuple haïtien et sa condition "pathétique ", la rencontre avec le matérialisme dialectique et son importance dans le cadre de l'action sociale souhaitée par le surréalisme (p. 30-32).

Pour envisager les ressorts de la révolte haïtienne de toute autre sorte que magiques, nous pouvons renvoyer aux lettres que Mabille écrivait entre janvier et avril 1946 à son supérieur Laugier (le directeur des relations culturelles à Paris), qui ne sont pas mentionnées dans Messagers de la tempête et qui pourtant correspondent parfaitement à la reconstruction du contexte révolutionnaire dans le témoignage de Bloncourt ${ }^{2}$. Par la formule suivante : « la révolution sociale n'est pas un but en soi, mais un moyen pour la libération de l'esprit humain... » (p. 32), Löwy titre la conclusion probablement la plus importante de ces événements pour indiquer l'écart absolu du surréalisme par rapport à la plupart des mouvements révolutionnaires et même par rapport à ces ethnologues qui, dans leurs recherches et classifications savantes, manquent parfois l'enjeu " anthropologique » par excellence, celui-là même qu'énonce Löwy.

\section{NOTES}

1. Vincent Bounoure, Le Surréalisme et les arts sauvages. Paris, L'Harmattan, $2001: 46$.

2. Rémy Laville, Pierre Mabille : un compagnon du surréalisme. Clermont-Ferrand, Presses de l'Université de Clermont-Ferrand, fascicule XVI, 1983 : 59-60. 


\section{AUTEURS}

ANDREA D'URSO

durso3@unisi.it 